\title{
Factores que explican las competencias emprendedoras
}

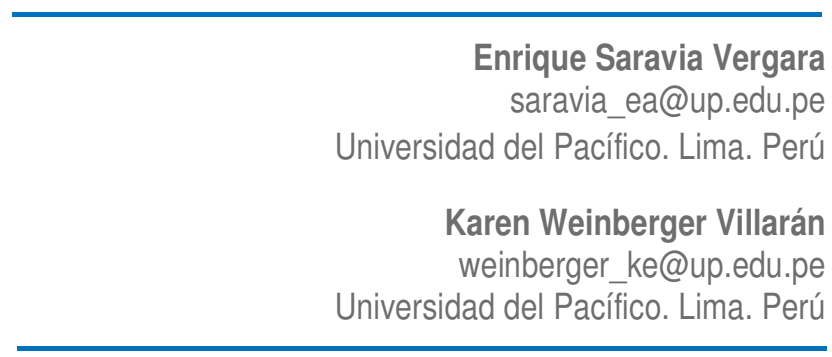

\section{Resumen}

El estudio presenta un análisis a nivel exploratorio de la evaluación de 74 Planes de Negocio, en el marco del curso Proyecto Empresarial en la Universidad del Pacífico, con el fin de identificar las competencias de emprendimiento de los estudiantes más relevantes según la opinión de los jurados especialistas, y su incidencia en la evaluación del Proyecto. El análisis factorial del estudio, a nivel exploratorio y confirmatorio con altos índices de confiabilidad y validez, identifica 4 conceptos vinculados a la evaluación de competencias: "aplicación de conocimientos", "comunicación", "manejo de información" y "visión empresarial, en lugar de las 7 dimensiones contenidas en el sistema de evaluación original: "Expresión Escrita", "Expresión Oral", "Creatividad", "Información", "Conceptos", "Razonamiento" y "Decisiones”. Un segundo hallazgo del estudio es que los alumnos emprendedores fueron mejor evaluados en las competencias de "comunicación" y "manejo de información", y peor evaluados en la "visión de negocios" y la "aplicación de conocimientos". Como tercer hallazgo, el análisis estructural demostró que las dimensiones "visión de negocios", "información" y "conocimiento" logran explicar la nota o evaluación final de los Planes de Negocio, mientras que las competencias de "comunicación" no influyen en la calificación de los jurados especialistas.

Palabras clave: Emprendimiento. Competencias emprendedoras. Planes de Negocio 


\section{Introducción}

La Universidad del Pacífico (Lima - Perú) líder en la formación de estudiantes de Administración de Empresas en el nivel de pregrado, promueve el desarrollo de habilidades emprendedoras y competencias gerenciales, como atributos fundamentales de su propuesta de valor.

A lo largo de todo el plan de estudios y de manera particular en el curso "Proyecto Empresarial", materia obligatoria del décimo ciclo de estudios, los estudiantes desarrollan grupalmente un plan de negocios. Este plan de negocios, basado en un modelo de negocio previamente validado en el mercado (a través de fuentes de investigación primaria y secundaria) debe demostrar ser económicamente rentable, operativamente viable, socialmente responsable y ambientalmente amigable.

Aproximadamente el $20 \%$ de esos proyectos se pone en marcha, ya sea con el modelo de negocios inicialmente propuesto o con algunas modificaciones. Si bien no todos los proyectos superan el "valle de la muerte". Una de las lecciones aprendidas es que la pasión por el proyecto, el entusiasmo, la perseverancia de los promotores y no tener miedo al fracaso, son factores claves para la implementación del mismo. Sin embargo, falta indagar acerca de las competencias que más influyen en la elaboración del proyecto, si están más vinculadas a los conocimientos técnicos o a otras competencias que puedan ser relevantes para medir el perfil del emprendedor. Como objetivo del artículo se plantea identificar las competencias más relevantes del perfil del emprendedor y medir su aporte en la elaboración de Planes de Negocio.

\section{Marco Teórico}

La palabra "emprendimiento" surge del término francés entrepreneur, que significa estar listo para tomar decisiones o iniciar algo (Rodriguez Ramirez, 2009). Por su parte la Real Academia Española, define al emprendedor o emprendedora, como la persona "que emprende con resolución acciones dificultosas o azarosas". Asimismo, define el verbo emprender como la acción de "acometer y comenzar una obra, un negocio, un empeño, especialmente si encierran dificultad o peligro". Es decir, el emprendimiento involucra dificultad y riesgo.

Para estudiar el emprendimiento hay que reconocer su naturaleza interdisciplinaria y su complejidad, identificándose cuatro perspectivas de pensamiento: la comportamental o de comportamiento, la psicológica o cognitiva, la económica y la de procesos (Rodríguez Ramírez, 2009). Desde estas perspectivas, el emprendedor tiene un perfil psicológico, desarrolla esquemas y conocimientos, pretende ser un dinamizador del desarrollo económico y se involucra en los procesos de emprendimiento que incluye actividades y funciones relacionadas a la percepción, aprovechamiento y evaluación de oportunidades.

El emprendedor es una persona con capacidad de crear, de llevar a cabo sus ideas y crear bienes y servicios, de asumir riesgos, capaz de "mirar" y "ver" su entorno y descubrir oportunidades de negocio. Posee iniciativa propia y sabe estructurar su proyecto, se comunica y conforma redes (Formichella, 2004). 
Otros autores, como Rodrigo Varela (Varela V., 2008) señalan que si bien no hay uniformidad en la definición de emprendedores, todo aquel que pone en marcha una empresa debe ser llamado empresario y no emprendedor. Para Varela, "El empresario es la persona o conjunto de personas capaces de percibir una oportunidad y ante ella formular, libre e independientemente, una decisión de consecución y asignación de los recursos naturales, financieros, tecnológicos y humanos necesarios para poner en marcha la empresa, que además de crear valor incremental para la economía, genera trabajo para él $y$, muchas veces, para otros. En este proceso de liderazgo creativo e innovador, el empresario invierte energía, dinero, tiempo y conocimientos, participa activamente en el montaje y operación de la empresa, arriesga sus recursos y su prestigio personal, y busca recompensas monetarias, personales y/o sociales y genera, con responsabilidad, bienestar social" (Varela V., 2008, pág. 126)

Hay características en común entre emprendedores o empresarios ellos que son: la capacidad para identificar oportunidades, la creatividad e innovación para satisfacer necesidades, la capacidad para conseguir y asignar los recursos escasos de manera eficiente, la capacidad para tomar decisiones y resolver problemas, así como la habilidad para trabajar en equipo y liderarlo hacia la consecución de objetivos comunes.

Una pregunta frecuente en las universidades acerca del tema de emprendimiento, es si los emprendedores nacen o se hacen. Reconociendo que la educación es capaz de modificar actitudes y comportamientos, entonces es posible afirmar que los alumnos aprenderán a ser emprendedores (Formichella, 2004).

Sin embargo, para Juan Jordano Pérez (2006) el emprendedor nace y no se hace, cuando indica que "el espíritu emprendedor dimana del genotipo del individuo. Se tiene espíritu emprendedor porque se es optimista a la vez que innovador y de forma equilibrada" (Pérez, 2006, pág. 428). Para Jordano, la tarea de la universidad es reconocer e identificar a los emprendedores, porque solo con emprendedores valdría la pena trabajar para convertirlos en empresarios.

Sea que el emprendedor nazca, o se haga, las universidades se han concentrado en la enseñanza de conocimientos de gestión y el desarrollo de habilidades emprendedoras en un contexto generalmente teórico, de modo que los alumnos necesitan complementar lo aprendido con su intuición u otras herramientas (Formichella, 2004).

El modelo de desarrollo de emprendedores implica un cambio de paradigma, del enfoque de desarrollar una idea hasta convertirla en un proyecto viable, al enfoque de desarrollar el talento emprendedor. La formación de emprendedores debe poner énfasis en la preparación de planes de negocio, a desarrollar en los emprendedores la capacidad para identificar oportunidades sobre la base de la observación, generar soluciones creativas y plantear prototipos (Vicens \& Grullon, 2011). En este sentido, el plan de negocios se convierte en un "instrumento" a través del cual se desarrollan habilidades emprendedoras (pasión, entusiasmo, perseverancia, coraje, capacidad para identificar oportunidades y asumir riesgos calculados) y competencias empresariales (planificar, organizar, dirigir, controlar, tomar decisiones, transformar recursos eficientemente, con una visión estratégica y de largo plazo). 
En la Universidad del Pacifico, a través del curso Proyecto Empresarial, se pretende evaluar las habilidades emprendedoras y las competencias gerenciales de los alumnos de la carrera de Administración de Empresas, del pregrado. Para ello se han identificado diversos criterios de evaluación, entre los que se encuentran no sólo el dominio de conceptos teóricos, sino también la creatividad, el razonamiento, la toma de decisiones y la expresión oral y escrita para comunicar el plan de negocios. En cada uno de estos criterios, también se han definido subcriterios que se evalúan mediante preguntas o "reactivos" como por ejemplo, identificar, aprovechar y evaluar oportunidades de negocio en la dimensión "creatividad"; recopilar, organizar e interpretar datos en la perspectiva "información"; estructura y redacción como "expresión escrita"; coherencia de ideas y entonación en la dimensión "expresión oral"; la identificación y consistencia de estrategias en la perspectiva "razonamiento", entre otros. Todas las preguntas, o criterios a evaluar, tienen el mismo peso relativo en la calificación final del plan de negocios, que comprende el trabajo escrito y la presentación y sustentación del mismo.

\section{Metodología}

El presente estudio se basó en utilizar las encuestas de evaluación del curso Proyecto Empresarial en la Universidad del Pacífico, compuesto por 20 preguntas agrupadas en las dimensiones: Expresión Escrita, Expresión Oral, Creatividad, Información, Conceptos, Razonamiento y Decisiones.

En el curso Proyecto Empresarial los alumnos desarrollan un Plan de Negocios durante un ciclo académico de 4 meses, asesorados por un profesor especialista en emprendimiento. Al final del ciclo, el desempeño de los estudiantes es medido por un Jurado Evaluador compuesto por 3 profesores de la facultad de Ciencias Empresariales. Estos docentes son, especialistas, a nivel táctico o estratégico, en marketing, recursos humanos, administración de operaciones, comercio internacional, contabilidad y finanzas, y en la industria o sector en el que se plantee el plan de negocios.

La evaluación considera la calidad del trabajo escrito, así como la presentación y sustentación del mismo. El trabajo escrito y la presentación final del plan de negocio, recibirán una calificación grupal, mientras que la sustentación del plan de negocios será calificada de manera independiente para cada alumno. La evaluación final se inicia con la calificación del trabajo escrito, para lo cual cada jurado debería haber revisado profundamente el plan de negocios. Luego se calificará la presentación del mismo y finalmente la sustentación de las decisiones tomadas durante el desarrollo del plan de negocios. Los jurados deberán calificar a cada uno de los alumnos empleando la hoja de evaluación que se encuentra en el anexo No. 1. Esta hoja contiene 20 criterios que deberán ser evaluados en una escala de 1 a 3 , y ponen una nota en una escala de 1 a 20.

En la tabla 1 se muestran las dimensiones y preguntas del cuestionario, en donde se puede contrastar que los criterios de evaluación coinciden con los conceptos identificados en la revisión de la literatura. 
Tabla 1. Definición de Variables

\begin{tabular}{|c|c|c|}
\hline $\begin{array}{l}\text { Dimensión } \\
\text { Del Cuestionario }\end{array}$ & Variable & Reactivo de Evaluación \\
\hline \multirow{2}{*}{ Expresión Escrita } & C01. Estructura & Coherencia de la estructura empleada \\
\hline & C02. Redacción & Redacción y estilo \\
\hline \multirow{4}{*}{$\begin{array}{c}\text { Expresión } \\
\text { Oral }\end{array}$} & C03. Coherencia & Coherencia de las ideas \\
\hline & C04. Entonación & Entonación y dicción \\
\hline & C05. Argumentos & $\begin{array}{l}\text { Inferencia de argumentos inductivos y } \\
\text { deductivos }\end{array}$ \\
\hline & C06. Convicción & $\begin{array}{l}\text { Prestar, sustentar y defender con convicción el } \\
\text { plan de negocios }\end{array}$ \\
\hline \multirow{6}{*}{ Creatividad } & C07. Idea Negocio & Detectar la oportunidad de negocio \\
\hline & C08. Evaluación & Evaluar la oportunidad de negocio \\
\hline & C09. Aprovechamiento & Aprovechar la oportunidad \\
\hline & C10. Soluciones & $\begin{array}{l}\text { Exploración de alternativas de solución: } \\
\text { creativas e innovadoras }\end{array}$ \\
\hline & C11. Presentación & Presentación del plan de negocio \\
\hline & C12. Convencimiento & Capacidad para vender la idea de negocio \\
\hline \multirow{3}{*}{ Información } & C13. Recopilación & Recopilar información \\
\hline & C14. Organización & Organizar y sistematizar información \\
\hline & C15. Interpretación & Analizar e interpretar la información \\
\hline \multirow{2}{*}{ Conceptos } & C16. Conocimiento & Conocimiento de conceptos \\
\hline & C17. Aplicación & Pertinencia y adecuada aplicación de conceptos \\
\hline \multirow{2}{*}{ Razonamiento } & C18. Estrategias & Identificación de estrategias \\
\hline & C19. Consistencia & Consistencia entre las estrategias planteadas \\
\hline Decisiones & C20. Toma de Decisiones & $\begin{array}{l}\text { Obtener conclusiones y tomar decisiones a } \\
\text { partir de un conjunto de información }\end{array}$ \\
\hline Evaluación & C21. NOTA & $\begin{array}{l}\text { Evaluación del Jurado conformado por } 3 \\
\text { profesores, en una escala de } 0 \text { a } 20 \text { (Variable } \\
\text { Dependiente) }\end{array}$ \\
\hline
\end{tabular}

Fuente: Formato de Encuesta del curso Proyecto Empresarial de la Universidad del Pacífico

Para el presente estudio se sistematizó la evaluación de 74 Planes de Negocio, correspondientes a tres ciclos académicos de estudio: abril-julio 2011, agosto-diciembre 2011 y abril-julio 2012. De esta forma, la investigación corresponde a un estudio 
concluyente al grupo de análisis, dado que el instrumento se aplicó a nivel de censo a todos los proyectos del período de análisis.

En particular, las evaluaciones de los Planes de Negocio se procesaron mediante las siguientes técnicas estadísticas:

a) Análisis de Estadísticas Descriptivas.

b) Análisis Factorial Exploratorio, mediante el análisis por componentes principales

c) Análisis Factorial Confirmatorio, mediante el modelo de medida de ecuaciones estructurales

d) Análisis de Ecuaciones Estructurales.

\section{Resultados}

De acuerdo a la metodología prevista, a continuación se resumen los principales resultados en cada uno de las técnicas estadísticas utilizadas:

\section{a) Análisis de Estadísticas Descriptivas}

El análisis estadístico se realizó con el software SPSS y, en esta etapa, se calcularon la media (M) como medida de tendencia central y la desviación estándar (DS) como medida de dispersión o de variabilidad. A continuación se detallan los resultados:

Tabla 2. Resultados descriptivos

\begin{tabular}{lccccc}
\hline & $\mathrm{N}$ & Mínimo & Máximo & Media & Desv. típ. \\
\hline C01. Expresión Escrita - Estructura & 74 & 1,0 & 3,0 & 2,662 &, 5241 \\
C02. Expresión Escrita - Redacción & 74 & 1,0 & 3,0 & 2,561 &, 6135 \\
C03. Expresión Oral - Coherencia & 74 & 1,0 & 3,0 & 2,507 &, 5395 \\
C04. Expresión Oral - Entonación & 74 & 1,0 & 3,0 & 2,797 &, 4373 \\
C05. Expresión Oral - Argumentos & 74 & 1,0 & 3,0 & 2,351 &, 5952 \\
C06. Expresión Oral - Convicción & 74 & 1,0 & 3,0 & 2,568 &, 6374 \\
C07. Creatividad - Idea Negocio & 74 & 1,0 & 3,0 & 2,797 &, 4294 \\
C08. Creatividad - Evaluación & 74 & 1,0 & 3,0 & 2,358 &, 6055 \\
C09. Creatividad - Aprovechamiento & 74 & 1,0 & 3,0 & 2,446 &, 5827 \\
C10. Creatividad - Soluciones & 74 & 1,0 & 3,0 & 2,236 &, 6984 \\
C11. Creatividad - Presentación & 74 & 1,0 & 3,0 & 2,682 &, 5400 \\
C12. Creatividad - Convencimiento & 74 & 1,0 & 3,0 & 2,426 &, 6500 \\
C13. Información - Recopilación & 74 & 1,0 & 3,0 & 2,527 &, 6405 \\
C14. Información - Organización & 74 & 1,0 & 3,0 & 2,412 &, 6892 \\
C15. Información - Interpretación & 74 & 1,0 & 3,0 & 2,345 &, 6244 \\
C16. Conceptos - Conocimiento & 74 & 1,0 & 3,0 & 2,155 &, 5964 \\
C17. Conceptos - Aplicación & 74 & 1,0 & 3,0 & 2,108 &, 6263 \\
C18. Razonamiento - Estrategias & 74 & 1,0 & 3,0 & 2,453 &, 5920 \\
C19. Razonamiento - Consistencia & 74 & 1,0 & 3,0 & 2,297 &, 6668 \\
C20. Decisiones - Toma Decisiones & 74 & 1,0 & 3,0 & 2,547 &, 5862 \\
NOTA & 74 & 11,50 & 20,00 & 16,3547 & 1,93738 \\
\hline N válido (según lista) & 74 & & & & \\
\hline
\end{tabular}

Fuente: Evaluación de alumnos en el curso Proyecto Empresarial de la Universidad del Pacífico, ciclos 2011-I, 2011-II y 2012-I. Elaboración: software SPSS 
Tal como se puede apreciar, las cinco competencias mejor calificadas por los docentes fueron las siguientes:

$\begin{array}{lll}\checkmark & \text { Expresión Oral - Entonación } & \mathrm{M}=2.797 \\ \checkmark & \text { Creatividad - Idea Negocio } & \mathrm{M}=2.797 \\ \checkmark & \text { Creatividad - Presentación } & \mathrm{M}=2.682 \\ \checkmark & \text { Expresión Escrita - Estructura } & \mathrm{M}=2.662 \\ \checkmark & \text { Expresión Oral - Convicción } & \mathrm{M}=2.568\end{array}$

Por el contrario, según la evaluación, las cinco competencias con menor desempeño por parte de los alumnos fueron las siguientes:

$\begin{array}{lll}\checkmark \text { Conceptos - Aplicación } & M=2.108 \\ \checkmark \text { Conceptos - Conocimiento } & \mathrm{M}=2.155 \\ \checkmark \text { Creatividad - Soluciones } & \mathrm{M}=2.236 \\ \checkmark & \text { Razonamiento - Consistencia } & \mathrm{M}=2.297 \\ \checkmark & \text { Información - Interpretación } & \mathrm{M}=2.446\end{array}$

Estos resultados a nivel descriptivo y como una primera aproximación, muestran que los alumnos destacan más por competencias de expresión oral y escrita y creatividad: "Entonación y dicción", "Detectar la oportunidad de negocio", "Presentación del plan de negocio", "Coherencia de la estructura empleada" y "Prestar, sustentar y defender con convicción el plan de negocios", en ese orden; mientras que las principales debilidades son: "Pertinencia y adecuada aplicación de conceptos", "Conocimiento de conceptos", "Exploración de alternativas de solución: creativas e innovadoras", "Consistencia entre las estrategias planteadas" y "Analizar e interpretar la información". En este análisis llama la atención que los alumnos destaquen por su expresión oral y escrita, además de su creatividad para detectar oportunidades de negocio, y tienen más problemas vinculados al conocimiento y la aplicación de conceptos.

\section{b) Análisis de Confiabilidad y Validez Convergente}

Al plantear el coeficiente Alfa de Crobanch y el análisis factorial por principales componentes en el software SPSS, no se lograron niveles adecuados de confiabilidad y de validez en ninguna de las dimensiones del instrumento de evaluación de alumnos, razón por la cual se procesaron todos los datos juntos, es decir, sin agruparlos en las dimensiones propuestas por el cuestionario de evaluación.

El análisis factorial conjunto determinó una estructura subyacente a la propuesta por el instrumento de evaluación; definió 4 dimensiones subyacentes bajo el método de rotación de factores VARIMAX. A continuación se resumen la reestructuración de variables en nuevas dimensiones subyacentes: 
Tabla 3. Estructura Factorial resultante del Análisis Factorial

\begin{tabular}{|c|c|c|c|c|}
\hline \multirow{2}{*}{ Variable } & \multicolumn{4}{|c|}{ Componente } \\
\hline & 1 & 2 & 3 & 4 \\
\hline C01. Expresión Escrita - Estructura &, 844 & ,257 &, 125 &, 064 \\
\hline C02. Expresión Escrita - Redacción &, 663 & ,177 & ,161 & ,006 \\
\hline C03. Expresión Oral - Coherencia &, $\mathbf{3 6 0}$ &, 462 & ,210 & ,205 \\
\hline C04. Expresión Oral - Entonación &,- 181 & ,723 & 296 & ,026 \\
\hline C05. Expresión Oral - Argumentos &, 564 & ,104 & ,319 & ,174 \\
\hline C06. Expresión Oral - Convicción & ,236 & ,715 & ,091 & ,317 \\
\hline C07. Creatividad - Idea Negocio &,- 108 & ,045 & ,082 & ,715 \\
\hline C08. Creatividad - Evaluación &, 456 &, 161 & ,247 &, 571 \\
\hline C09. Creatividad - Aprovechamiento &, 167 & ,215 &, 591 &, 501 \\
\hline C10. Creatividad - Soluciones &, 213 & ,202 & ,162 & ,716 \\
\hline C11. Creatividad - Presentación & ,636 &, 441 &, 010 & ,302 \\
\hline C12. Creatividad - Convencimiento &, 383 &, 660 &, 020 & ,390 \\
\hline C13. Información - Recopilación &, 532 & ,072 &, 431 & ,084 \\
\hline C14. Información - Organización & ,906 &, $\mathbf{1 1 8}$ & ,131 &, 020 \\
\hline C15. Información - Interpretación & ,669 & ,111 & ,316 & ,300 \\
\hline C16. Conceptos - Conocimiento & ,231 &, 151 & ,762 & ,199 \\
\hline C17. Conceptos - Aplicación & ,240 & ,227 &, 814 &, $\mathbf{1 5 1}$ \\
\hline C18. Razonamiento - Estrategias &, 384 & ,619 & ,165 &,- 076 \\
\hline C19. Razonamiento - Consistencia & ,356 & ,409 & ,436 & ,018 \\
\hline C20.Decisiones - Toma Decisiones &, 574 &, 078 & ,327 &, 507 \\
\hline
\end{tabular}

Fuente: Evaluación de alumnos en el curso Proyecto Empresarial de la Universidad del Pacífico, ciclos 2011-I, 2011-II y 2012-I. Elaboración: software SPSS

A continuación se resumen los principales argumentos que explican la pertinencia de aplicar el Análisis Factorial conjunto con las 20 variables:

(i) A nivel general, con las 20 variables, el test de confiabilidad determinó una alta confiabilidad de los datos, con un índice Alfa de Cronbach de 0.925, muy superior al límite inferior de 0.70 comúnmente aceptado.

(ii) El análisis factorial logró un alto nivel de ajuste, un índice KMO (KaiserMeyer-Olkin) de 0.838, "muy bueno".

(iii) Se identificó una estructura adyacente de 4 dimensiones estadísticamente significativa, con una validez aceptable de $63.03 \%$ de varianza explicada, superior al límite inferior de $55 \%$ comúnmente aceptado.

(iv) El análisis factorial de cada dimensión también permitió comprobar la confiabilidad y validez de cada una de las nuevas dimensiones. En estos análisis, por confiabilidad y validez se tuvieron que eliminar dos variables: "C04. Expresión Oral - Entonación" y "C07. Creatividad - Idea Negocio". En la Tabla 6 se pueden apreciar las comunalidades de las variables y la confiabilidad y validez de las nuevas dimensiones subyacentes.

(v) Las 4 nuevas dimensiones permiten identificar 4 nuevos conceptos, es decir, 
es posible que las variables agrupadas en cada uno de los nuevos conceptos permiten interpretar el concepto que representan. En particular, se identificaron y se definieron los siguientes términos asociados a los nuevos conceptos:

Tabla 4. Definición de nuevas dimensiones

\begin{tabular}{|c|c|c|}
\hline Nueva Dimensión & Definición & Variables \\
\hline FACTOR 1 & Manejo de Información & $\begin{array}{l}\mathrm{C} 01, \mathrm{C} 02, \mathrm{C} 05, \mathrm{C} 11, \mathrm{C} 13, \\
\mathrm{C} 14, \mathrm{C} 15, \mathrm{C} 20\end{array}$ \\
\hline FACTOR 2 & Comunicación & $\mathrm{C} 03, \mathrm{C} 04, \mathrm{C} 06, \mathrm{C} 12, \mathrm{C} 18$ \\
\hline FACTOR 3 & $\begin{array}{l}\text { Aplicación de } \\
\text { conocimientos }\end{array}$ & $\mathrm{C} 07, \mathrm{C} 08, \mathrm{C} 10$ \\
\hline FACTOR 4 & Visión empresarial & $\mathrm{C} 09, \mathrm{C} 16, \mathrm{C} 17, \mathrm{C} 19$ \\
\hline
\end{tabular}

Fuente: basado en resultados de la Evaluación de alumnos en el curso Proyecto Empresarial de la Universidad del Pacífico, ciclos 2011-I, 2011-Il y 2012-I. Elaboración propia en base a reportes del software SPSS

(vi) La evaluación de resultados descriptivos, en cada nueva dimensión, fueron los siguientes:

\author{
$\checkmark \quad$ FACTOR 2: Comunicación \\ $\checkmark$ FACTOR 1: Manejo de Información \\ $\checkmark$ FACTOR 4: Visión \\ $\checkmark \quad$ FACTOR 3: Aplicación Conocimientos
}

Evaluación prom. $=2.550$

Evaluación prom. $=2.511$

Evaluación prom. $=2.464$

Evaluación prom. $=2.252$

Estos resultados, confirman los indicios encontrados en el análisis preliminar por variables.

\section{c) Análisis Factorial Confirmatorio}

Con el fin de comprobar la Validez Convergente de dimensiones, a nivel confirmatorio, se planteó un Modelo de Medida, el cual fue procesado en el software Lisrel. A continuación se muestra el diagrama de path de dicho modelo (Gráfico 1), los índices de ajuste del modelo (Tabla 5) y los principales parámetros del modelo en forma conjunta a los resultados del análisis de confiabilidad y el análisis factorial (Tabla 6): 
Gráfico 1. Diagrama Path del Modelo de Medida (Validez Convergente Confirmatorio)

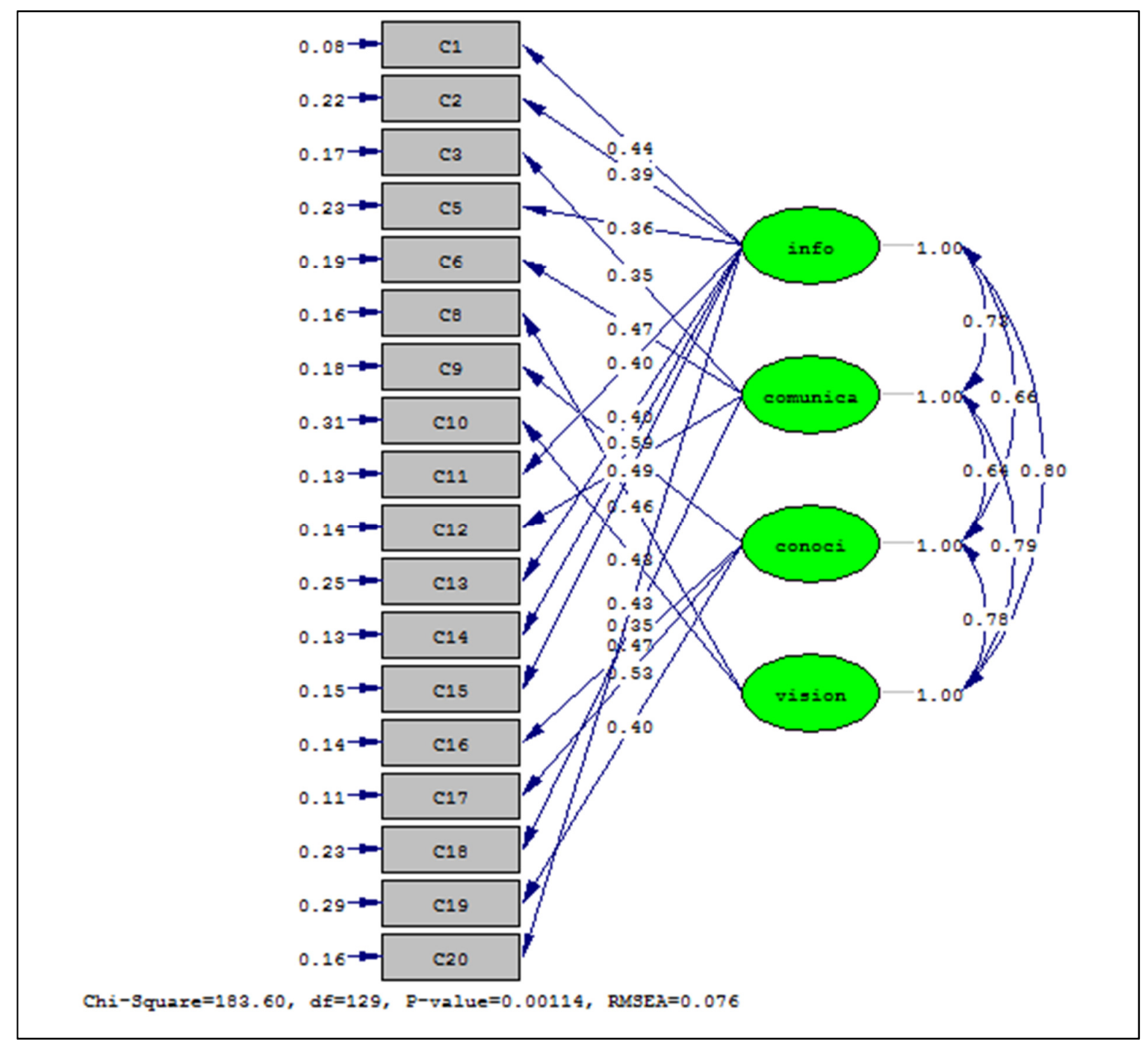

Fuente: datos de la Evaluación de alumnos en el curso Proyecto Empresarial de la Universidad del Pacífico, ciclos 2011-I, 2011-II y 2012-I. Elaboración: software LISREL 
Tabla 5. Índices de Ajuste del Modelo de Medida

\begin{tabular}{|c|c|c|}
\hline Tipo de Índice / Índice & Notación & Criterio de Validación \\
\hline \multicolumn{3}{|c|}{ Índices de Bondad de Ajuste Globales } \\
\hline$\chi=183.60 ; \mathrm{gl}=129$ & $\chi$ & \\
\hline $\mathrm{p}=0.00013$ & $\mathrm{p}$ & $\mathrm{P}<0.05$ \\
\hline$\chi / \mathrm{gl}=1.42$ & $\chi / \mathrm{gl}$ & $\chi / \mathrm{gl}<3$ \\
\hline \multicolumn{3}{|c|}{ Índices de Bondad de Ajuste Absoluto } \\
\hline $\mathrm{GFI}=0.78$ & GFI & GFI $>0.90$ \\
\hline $\mathrm{AGFI}=0.71$ & AGFI & AGFI $>0.90$ \\
\hline RMSEA $=0.076$ & RMSEA & $\begin{array}{c}\text { RMSEA }<0.08 \\
\text { Rechazar: RMSEA }>0.10\end{array}$ \\
\hline $\mathrm{RMR}=0.03$ & RMR & $\mathrm{RMR}<0.05$ \\
\hline $\mathrm{SRMR}=0.077$ & SRMR & SRMR $<0.05$ \\
\hline \multicolumn{3}{|c|}{ Índices de Bondad de Ajuste de Incremento } \\
\hline $\mathrm{NFI}=0.91$ & NFI & $\mathrm{NFI}>0.90$ \\
\hline $\mathrm{NNFI} / \mathrm{TLI}=0.96$ & NNFI / TLI & $\mathrm{NNFI}>0.90$ \\
\hline $\mathrm{CFI}=0.97$ & CFI & $\mathrm{CFI}>0.90$ \\
\hline $\mathrm{IFI}=0.97$ & IFI & IFI $>0.90$ \\
\hline $\mathrm{RFI}=0.89$ & RFI & RFI $>0.90$ \\
\hline \multicolumn{3}{|c|}{ Índices de Bondad de Ajuste de Parsimonia } \\
\hline PNFI $=0.76$ & PNFI & PNFI $>0.90$ \\
\hline PGFI $=0.59$ & PGFI & PGFI $>0.90$ \\
\hline
\end{tabular}

Fuente: datos de la Evaluación de alumnos en el curso Proyecto Empresarial de la Universidad del Pacífico, ciclos 2011-I, 2011-II y 2012-I. Elaboración propia en base a reporte del software LISREL. 
Tabla 6. Confiabilidad y Validez Convergente

\begin{tabular}{|c|c|c|c|c|c|c|c|}
\hline \multirow{3}{*}{ Dimensión } & \multirow{3}{*}{ Variable } & \multirow{3}{*}{$\begin{array}{c}\begin{array}{c}\text { Confiabi- } \\
\text { lidad }\end{array} \\
\begin{array}{c}\text { Alfa de } \\
\text { Cronbach } \\
(70 \%)\end{array}\end{array}$} & \multicolumn{5}{|c|}{ Validez Convergente } \\
\hline & & & \multicolumn{3}{|c|}{$\begin{array}{c}\text { Exploratorio } \\
\text { (Análisis Factorial) }\end{array}$} & \multicolumn{2}{|c|}{$\begin{array}{l}\text { Confirmatorio } \\
\text { (Modelo de } \\
\text { Medida) }\end{array}$} \\
\hline & & & $\begin{array}{l}\text { KMO } \\
(\mathbf{5 0 \%})\end{array}$ & $\begin{array}{c}\text { AVE } \\
(\mathbf{5 5 \%})\end{array}$ & $\begin{array}{c}\text { Comuna- } \\
\text { - } \\
\text { nalidades } \\
(\mathbf{0 . 5 0 )}\end{array}$ & $\begin{array}{c}\text { Signif. } \\
\mathbf{t} \\
(1.96)\end{array}$ & $\begin{array}{c}\text { Coefi- } \\
\text { ciente } \\
\mathbf{R 2} \\
(\mathbf{0 . 3 0}) \\
\end{array}$ \\
\hline \multirow{8}{*}{$\begin{array}{l}\text { Manejo de } \\
\text { informa- } \\
\text { ción }\end{array}$} & $\begin{array}{l}\text { C01. Coherencia de la estructura } \\
\text { empleada }\end{array}$ & \multirow{8}{*}{$89.5 \%$} & \multirow{8}{*}{$89.1 \%$} & \multirow{8}{*}{$58.6 \%$} & 0.734 & 8.70 & 0.71 \\
\hline & C02. Redacción y estilo & & & & 0.486 & 5.98 & 0.41 \\
\hline & $\begin{array}{r}\text { C05. Inferencia de argumentos } \\
\text { inductivos y deductivos }\end{array}$ & & & & 0.423 & 5.47 & 0.36 \\
\hline & C11. Presentación del plan de negocio & & & & 0.575 & 7.24 & 0.55 \\
\hline & C13. Recopilar información & & & & 0.450 & 5.73 & 0.39 \\
\hline & $\begin{array}{c}\text { C14. Organizar y sistematizar } \\
\text { información }\end{array}$ & & & & 0.780 & 8.85 & 0.72 \\
\hline & $\begin{array}{c}\text { C15. Analizar e interpretar la } \\
\text { información }\end{array}$ & & & & 0.656 & 7.74 & 0.61 \\
\hline & $\begin{array}{l}\text { C20. Obtener conclusiones y tomar } \\
\text { decisiones a partir de un conjunto de } \\
\text { información }\end{array}$ & & & & 0.589 & 7.15 & 0.54 \\
\hline \multirow{5}{*}{$\begin{array}{l}\text { Comunica- } \\
\text { ción }\end{array}$} & C03. Coherencia de las ideas & \multirow{5}{*}{$\mathbf{7 8 . 7 \%}$} & \multirow{5}{*}{$\mathbf{7 7 . 4 \%}$} & \multirow{5}{*}{$61.1 \%$} & 0.551 & 5.68 & 0.41 \\
\hline & C04. Entonación y dicción & & & & - & - & - \\
\hline & $\begin{array}{l}\text { C06. Prestar, sustentar y defender con } \\
\text { convicción el plan de negocios }\end{array}$ & & & & 0.671 & 6.82 & 0.54 \\
\hline & $\begin{array}{l}\text { C12. Capacidad para vender la idea de } \\
\text { negocio }\end{array}$ & & & & 0.712 & 7.81 & 0.66 \\
\hline & C18. Identificación de estrategias & & & & 0.511 & 5.21 & 0.36 \\
\hline \multirow{4}{*}{$\begin{array}{l}\text { Aplicación } \\
\text { de conoci- } \\
\text { mientos }\end{array}$} & C09. Aprovechar la oportunidad & \multirow{4}{*}{$80.1 \%$} & \multirow{4}{*}{$74.7 \%$} & \multirow{4}{*}{$63.3 \%$} & 0.568 & 6.16 & 0.46 \\
\hline & C16. Conocimiento de conceptos & & & & 0.691 & 7.47 & 0.61 \\
\hline & $\begin{array}{l}\text { C17. Pertinencia y adecuada aplicación } \\
\text { de conceptos }\end{array}$ & & & & 0.787 & 8.33 & 0.71 \\
\hline & $\begin{array}{l}\text { C19. Consistencia entre las estrategias } \\
\text { planteadas }\end{array}$ & & & & 0.487 & 5.25 & 0.35 \\
\hline \multirow{3}{*}{ Visión } & C07. Detectar la oportunidad de negocio & \multirow{3}{*}{$62.7 \%$} & \multirow{3}{*}{$\mathbf{5 0 . 0 \%}$} & \multirow{3}{*}{$73.1 \%$} & - & - & - \\
\hline & C08. Evaluar la oportunidad de negocio & & & & $\mathbf{0 . 7 3 1}$ & 6.42 & 0.57 \\
\hline & $\begin{array}{l}\text { C10. Exploración de alternativas de } \\
\text { solución: creativas e innovadoras }\end{array}$ & & & & 0.731 & 5.18 & 0.37 \\
\hline
\end{tabular}

Fuente: datos de la Evaluación de alumnos en el curso Proyecto Empresarial de la Universidad del Pacífico, ciclos 2011-I, 2011-II y 2012-I. Elaboración propia en base a reportes de los software SPSS y LISREL.

\section{d) Modelo Estructural}

Se planteó un modelo estructural utilizando el software LISREL, con el fin de tratar de explicar la evaluación de los profesores miembros del jurado, en función de los criterios de evaluación: información, comunicación, conocimientos y visión. En concreto, el modelo se ajustó a la siguiente ecuación estructural:

\section{Ecuación Estructural:}

Nota $=0.29 *$ info $+0.047 *$ conoci $+0.52 *$ visión, Errorvar. $=0.37$

R2 $=0.63$
(0.18)
(0.20)
(0.28) (0.085)
1.63
0.23
$\begin{array}{ll}1.83 & 4.42\end{array}$ 
Si bien ninguno de las dimensiones resulta estadísticamente significativa para explicar la nota de evaluación de los estudiantes (valores de significancia estadística de 1.63, 0.23 y 1.83; todos son menores al valor mínimo requerido de $\mathrm{t}=1.96$ para definir una relación significativa al 95\% de confianza), en conjunto las dimensiones "visión", "información" y "conocimiento" lograron explicar la nota con un índice de correlación r2 de 0.63. El modelo estructural no incluyó a la dimensión "comunicación".

En el Gráfico 2 se muestra la representación gráfica del modelo estructural, y en la Tabla 7 se presentan los índices de ajuste de dicho modelo:

Gráfico 2. Modelo Estructural

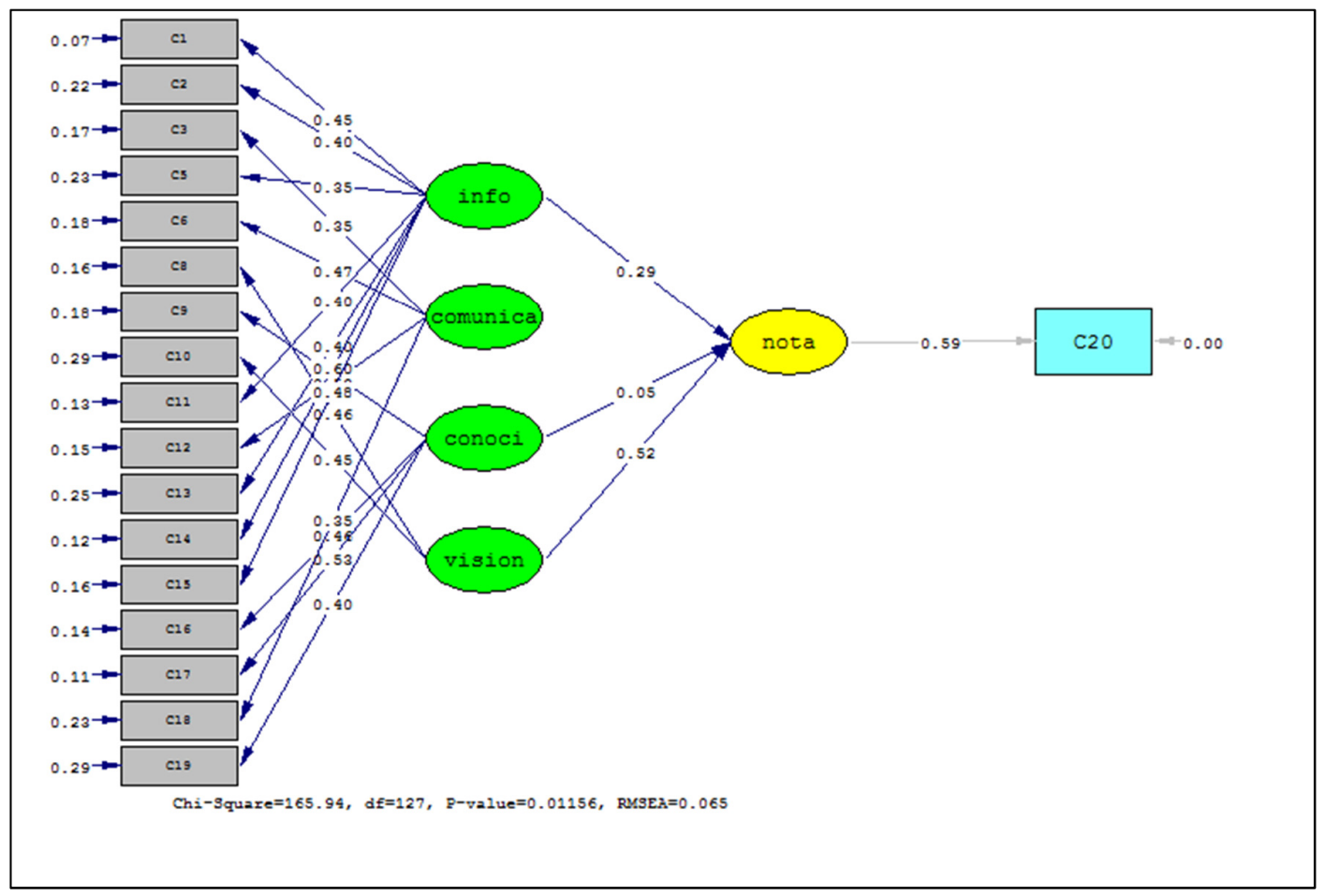

Fuente: datos de la Evaluación de alumnos en el curso Proyecto Empresarial de la Universidad del Pacífico, ciclos 2011-I, 2011-II y 2012-I. Elaboración: software LISREL 
Tabla 7. Índices de Ajuste del Modelo Estructural

\begin{tabular}{|c|c|c|}
\hline Tipo de Indice / Indice & Notación & Criterio de Validación \\
\hline \multicolumn{3}{|c|}{ Índices de Bondad de Ajuste Globales } \\
\hline$\chi=165.94 ; \mathrm{gl}=127$ & $\mathrm{X}$ & \\
\hline $\mathrm{p}=0.01156$ & $\mathrm{P}$ & $\mathrm{P}<0.05$ \\
\hline$\chi / \mathrm{gl}=1.31$ & $\chi / \mathrm{gl}$ & $\chi / \mathrm{gl}<3$ \\
\hline \multicolumn{3}{|c|}{ Índices de Bondad de Ajuste Absoluto } \\
\hline $\mathrm{GFI}=0.80$ & GFI & GFI $>0.90$ \\
\hline $\mathrm{AGFI}=0.73$ & AGFI & AGFI $>0.90$ \\
\hline RMSEA $=0.065$ & RMSEA & $\begin{array}{c}\text { RMSEA }<0.08 \\
\text { Rechazar: } R M S E A>0.10\end{array}$ \\
\hline $\mathrm{RMR}=0.029$ & RMR & $\mathrm{RMR}<0.05$ \\
\hline $\mathrm{SRMR}=0.077$ & SRMR & SRMR $<0.05$ \\
\hline \multicolumn{3}{|c|}{ Índices de Bondad de Ajuste de Incremento } \\
\hline $\mathrm{NFI}=0.91$ & NFI & $\mathrm{NFI}>0.90$ \\
\hline $\mathrm{NNFI} / \mathrm{TLI}=0.96$ & NNFI / TLI & $\mathrm{NNFI}>0.90$ \\
\hline $\mathrm{CFI}=0.97$ & CFI & $\mathrm{CFI}>0.90$ \\
\hline $\mathrm{IFI}=0.97$ & IFI & IFI $>0.90$ \\
\hline $\mathrm{RFI}=0.89$ & RFI & RFI $>0.90$ \\
\hline \multicolumn{3}{|c|}{ Índices de Bondad de Ajuste de Parsimonia } \\
\hline PNFI $=0.76$ & PNFI & PNFI $>0.90$ \\
\hline PGFI $=0.59$ & PGFI & PGFI $>0.90$ \\
\hline
\end{tabular}

Fuente: datos de la Evaluación de alumnos en el curso Proyecto Empresarial de la Universidad del Pacífico, ciclos 2011-I, 2011-II y 2012-I. Elaboración propia en base a reporte del software LISREL 


\section{Conclusiones y Recomendaciones}

La valoración de los criterios de evaluación de los profesores presentó altos índices de confiabilidad y validez, lo que demuestra coherencia en la evaluación de criterios por parte de los profesores miembros del jurado. Los estudiantes del curso Proyecto Empresarial, de los ciclos académicos abril - julio 2011, agosto - diciembre 2011 y abril - julio 2012 mostraron ser más competentes en "comunicación" y "manejo de información", que en "visión" y "aplicación de conocimientos". Este hallazgo obliga a evaluar qué está pasando en el proceso de formación y qué debería hacerse para reforzar en el futuro las técnicas y aspectos pedagógicos vinculados a la visión de negocios y la aplicación de conocimientos de gestión, propios de la carrera profesional de los estudiantes. El conocimiento de la teoría, la pertinencia en la aplicación de conceptos, el análisis y la interpretación de la información recopilada, así como la consistencia de las estrategias plateadas para dar solución a los problemas de manera creativa, son aspectos que deberán ser reforzados a través de los procesos de aprendizaje, ya sea en Proyecto Empresarial, o en las asignaturas previas al mismo. Esto amerita una revisión de los contenidos, metodologías y procesos de aprendizaje del plan de estudios de administración. Por otro lado, el Taller de Presentaciones Efectivas, llevado a cabo por el equipo de psicólogos del área de Gestión del Aprendizaje, paralelo al desarrollo del curso, parece que está cumpliendo con el objetivo de mejorar las competencias blandas de los alumnos.

Otro hallazgo importante del estudio es la presencia de cuatro conceptos de evaluación por parte del jurado: i) la visión de negocios para detectar, aprovechar y evaluar oportunidades de negocio ii) las competencias vinculadas al manejo de información iii) la aplicación de conocimientos y iv). la comunicación oral y escrita. En esta línea, el cuestionario podría reestructurarse en el futuro en estas dimensiones, en lugar del cuestionario actual que presenta veinte criterios de evaluación, algunos de los cuales parecen repetirse. Cabe señalar que estos cuatro criterios están directamente vinculados a las habilidades emprendedoras y/o competencias empresariales necesarias para la puesta en marcha de un emprendimiento. Estas son: i) encontrar una oportunidad, ii) analizar la información para proponer un modelo de negocio y desarrollar un plan de negocio que demuestre ser viable, rentable y escalable, iii) aplicar los conocimientos para escoger las mejores estrategias y iv) tener el talento necesario para comunicar y vender la idea de negocio a potenciales inversionistas y clientes.

Durante el periodo de evaluación la expresión de los profesores es más sensible a la "visión", dimensión relacionada a la identificación y evaluación de oportunidades de negocio, y explorar alternativas de solución creativas e innovadoras. En menor medida, el "el manejo de información" y los "conocimientos" influyen en la nota. Por el contrario, la "comunicación" no mostró relación con la nota de evaluación de los jurados.

Si bien el análisis se ha orientado a la "validación" de los criterios de evaluación, cabe reflexionar sobre la "valoración" de los criterios mejor y peor evaluados por parte de los jurados. Al ser docentes especialistas en las diversas áreas funcionales y sectores económicos - tanto a nivel táctico como estratégico- es posible que sus expectativas en cuanto a la evaluación de su materia o expertise sean altas, por lo que la calificación en "visión" y "aplicación de conocimientos", en términos relativos es baja. En diversas ocasiones se ha podido observar que durante las sustentaciones los jurados exigen a 
los alumnos un nivel de profundidad y detalle en aspectos teóricos vinculados a su experiencia que va más allá de la evaluación del plan de negocios o aspectos vinculados al mismo. Esta exigencia académica dio lugar a una reestructuración en la asignatura. Hoy, durante el desarrollo del curso, se toman cinco controles de lectura para refrescar o reforzar los conocimientos teóricos y la aplicación de los mismos a los planes de negocio, en base a los hallazgos de esta investigación. Por otro lado, una generación de estudiantes con mayor facilidad para acceder a las tecnologías de la información y las comunicaciones, podría sorprender y superar las expectativas de los docentes, que no son nativos digitales. En este sentido, las dimensiones "comunicación" y "manejo de información" podrían estar mejor calificadas, en base a las expectativas de los docentes, más que al desempeño real de los estudiantes, en comparación con sus pares u otras asignaturas del plan de estudios.

En resumen, es recomendable revisar los veinte criterios de evaluación, la valorización de cada uno de ellos y su peso relativo en la calificación final del curso. Valdría la pena estudiar la posibilidad de reducir el número de criterios, lo que facilitaría la calificación para los jurados y establecer un peso relativo para cada criterio, en función a los objetivos de aprendizaje o competencias a desarrollar establecidos en el plan de estudios vigente.

Finalmente, se recomienda revisar diversos modelos educativos para el desarrollo de habilidades (actitudes) emprendedoras y competencias (capacidades) empresariales pues no existe una solución única aplicable a todos los grupos (Gibb, Allan, 2011). Por otro lado, comenzar a medir la "intención empresarial" (Yvon Gasse \& Maripier Tremblay, 2011) de los estudiantes de la carrera de administración de la Universidad del Pacífico, también podría dar lugar a cambios en el contenido, la metodología y criterios de evaluación de los estudiantes del curso Proyecto Empresarial. Finalmente, evitemos confundir la educación para la creación de nuevas empresas, con la educación para el desarrollo de las pequeñas y medianas empresas (Solomon, George, 2011). A través las incubadoras de empresas, como es el caso de Emprende UP, el Centro de Emprendimiento de la Universidad del Pacífico, se pueden generan estímulos positivos para la puesta en marcha de los planes de negocio desarrollados en el curso Proyecto Empresarial.

\section{Referencias bibliográficas}

Formichella, M. M. (2004). El concepto de emprendimiento y su relación con la educación, el empleo y el desarrollo local. Recuperado el Enero de 2012, de Instituto Nacional de Tecnología Agropecuaria (INTA), Buenos Aires: https://emprendedorusach.files.wordpress.com/2009/06/el-concepto-deemprendimieto-y-su-relacion-con-la-educaion-el-empleo-y-el-desarrollo-local.pdf

Gibb, Allan. (2011). Espíritu empresarial: Soluciones únicas para ambientes únicos. En R. Varela, Desarrollo, Innovación y Cultura Empresarial (pág. 220). Cali: Universidad Icesi - Centro de Desarrollo del Espíritu Empresarial.

Pérez, J. J. (2006). Evolución del emprendedor, al empresario, a la empresa. Boletín de Estudios Económicos, Vol. LXI - No.189 , 413 - 430. 
Rodriguez Ramirez, A. (2009). Nuevas perspectivas para entender el emprendimiento empresarial. Pensamiento y Gestión, 26, 94-119.

Solomon, George. (2011). La confusión curricular entre la educación en espíritu empresarial y la gerencia de pequeñas empresas: una revisión de los principales libros de texto. En R. Varela V., Desarrollo, Innovación y Cultura Empresarial (pág. 220). Cali: Universidad Icesi - Centro de Desarrollo del Espíritu Empresarial.

Varela V., R. (2008). Innovación empresarial. Arte y ciencia en la creación de empresas. Bogotá - Colombia.: Pearson Educaciòn de Colombia Ltda.

Vicens, L., \& Grullon, S. (2011). Innovación y emprendimiento. Un modelo basado en el desarrollo del emprendedor. Recuperado el Agosto de 2011, de Banco Interamericano de Desarrollo, BID: http://www.iadb.org

Yvon Gasse \& Maripier Tremblay. (2011). Las intenciones de crear empresa: Un estudio multicultural entre estudiantes universitarios de cuatro países. En R. Varela V., Desarrollo, Innovación y Cultura Empresarial (pág. 220). Cali: Universidad ICESI Centro de Desarrollo del Espíritu Empresarial. 